\section{Grégoria Mer}

Spécialiste qualifiée en ODF Pratique privée
Adresse pour correspondance orthodontiechaville@gmail.com
Chers lecteurs,

Le numéro varia rassemble les articles divers, soumis à la rédaction, et qui n'ont pas pu trouver leur place dans un numéro à thème.

Celui-ci démarre par une approche fonctionnelle de notre métier proposée par Nicolas Fougeront et Marc Bert.

Nicolas Fougeront nous propose une analyse fine du contrôle sensorimoteur de la dimension verticale. Cette approche permet de comprendre ou d'émettre des hypothèses quant aux pathologies liées au sens vertical : respiration buccale, DAM, etc.

Le numéro se poursuit par un cas clinique proposé par le Docteur Marc Bert, qui explore les effets d'une interférence occlusale sur le fonctionnement du système manducateur. Cette interférence peut être à l'origine de douleurs musculaires (contracture du muscle ptérigoïdien latéral) pouvant être confondues avec les douleurs d'une névralgie faciale ; le diagnostic différentiel est alors difficile à réaliser.

Nous rentrons ensuite dans un domaine plus " mécanique " de notre activité d'orthodontiste.

Anne-Charlotte Scotti nous propose de comparer deux types de dispositifs d'ancrage pour la distalisation molaire, les mini-vis OBS de Chris Chang et les mini-plaques d'ancrage, avec leurs avantages et leurs inconvénients, le tout illustré par des cas cliniques.

Le Professeur Roland Benoît poursuit son travail présenté en 2018 (Rev Orthop Dento Faciale 2018;52,351-372) sur une analyse génétique et fonctionnelle. II nous présente des cas cliniques, de typologies variées étayant son propos. 


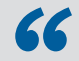

Un numéro qui présente une approche fonctionnelle puis un domaine plus "mécanique " de notre activité d'orthodontiste...
Nous republions et relisons ensuite avec plaisir, un article du Docteur Laurent Petitpas, dont l'iconographie avait été tronquée lors de la publication précédente (Rev Orthop Dento Faciale;53,297-315) et qui traite d'une reprise de traitement orthodontique, motivée par la présence de douleurs des articulations temporo-mandibulaires, analysée, avant, pendant et après traitement, par l'imagerie 3D.

Ce numéro s'achève bien sûr par une excellente revue de presse proposée par Hélène Desnoës.

Je remercie tous ceux qui ont participé à ce numéro, construit dans une période de confinement très particulière, et espère que vous prendrez plaisir à le lire. 